\title{
Dynamics of Apolar Guest Solubilized in Bile Salt Micelles: Photochemistry of Acenaphthylene as a Probe to Understand the Supramolecular Characteristics of the Aggregates
}

\author{
Nick Carter, Mahesh Pattabiraman*, Luis Albert Arias \\ Department of Natural Sciences, Western New Mexico University, Silver City, 88061, USA
}

\begin{abstract}
Product distribution resulting from the excited state reactivity of acenaphthylene is influenced predominantly by multiplicity of the excited state, mobility of the substrate in a given medium, and spatial constraints. The photochemistry of acenaphthylene solubilized by sodium cholate, a member of the atypical micelle forming molecules called bile salts, in water was investigated to understand the interior of the aggregates. Results indicate that the supramolecular characteristics of the aggregates change significantly with concentration which in turn affects the mobility of the encapsulated acenaphthylene guest leading to changes in product distribution from the reaction.
\end{abstract}

Keywords Bile salts, sodium cholate, micelles, complexation, photochemistry, acenaphthylene, excited state, supramolecular chemistry, green chemistry

\section{Introduction}

Micelles are supramolecular systems with tremendous applications in biotechnology, catalysis, medicine, nanotechnology, food science, environmental remediation, catalysis, and other areas of practical significance. ${ }^{1-8}$ Straight chain amphiphiles such as phospholipids, alkyl sulfonates, tetra alkyl ammonium salts, and alkyl polyglucosides have been well studied for their micelles. But bile salt micelles have remained relatively unexplored, ${ }^{9,10}$ especially for application in green chemistry as media to perform chemical transformations. ${ }^{11-13}$

Bile salts are produced in the liver of mammals and are understood to play important roles in digestion of fat. ${ }^{14-17}$ Bile salts are family of compounds with steroidal frame work and contain hydroxyl groups (two to three) and an acid group containing side-chain. The steroidal backbone of the molecule assumes a puckered shape in which the hydrophobic methyl groups occupy the convex side and the hydrophilic hydroxyl groups occupy the concave side (Figure 1) rendering the molecules amphiphilic. When dissolved in aqueous solutions at concentrations close to their formal CMC, the bile salts aggregate (Figure 2) to produce primary aggregates composed of 5-10 monomers ${ }^{18}$ in which the

* Corresponding author:

pattabiramanm@wnmu.edu (Mahesh Pattabiraman)

Published online at http://journal.sapub.org/chemistry

Copyright (C) 2012 Scientific \& Academic Publishing. All Rights Reserved convex side faces the interior of the aggregate and the concave side faces the aqueous phase. Furthermore, at higher concentrations the primary aggregates are known to aggregate further to yield larger loosely held structure called the secondary aggregates sustained by hydrogen-bonding between the hydroxyl groups of the bile salt and water molecules in the medium. ${ }^{18-20}$

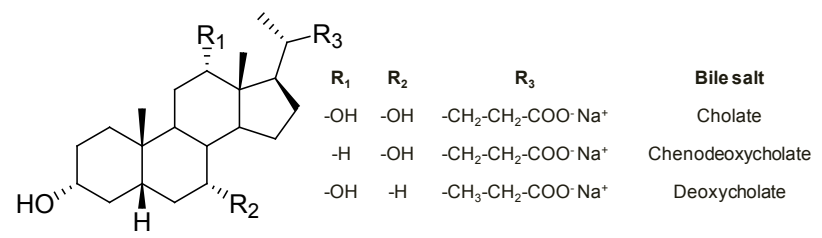

Figure 1. Chemical structure of bile salt monomers

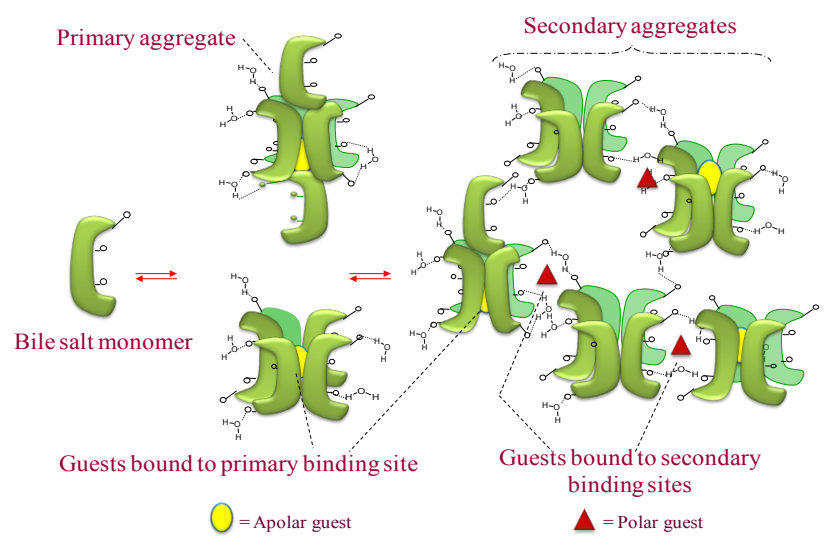

Figure 2. Representation of stepwise aggregation of bile salts in aqueous medium resulting in formation of primary and secondary aggregates 
The primary and secondary aggregates contain small pockets of empty space which are capable of accommodating guests of appropriate size and polarity. The pockets of space in the primary aggregates (primary binding sites) tend to be highly non-polar, and rigid, while the same in the secondary aggregates (secondary binding sites) tend to be more polar, less rigid, and relatively voluminous. The primary binding sites of bile salt micelles are excellent binding sites for nonpolar guest molecules such as polyaromatic hydrocarbons while the secondary aggregates accommodate large, polar guest molecules. Unlike the well-studied long chain micelles, bile salt micelles exhibits a much more complex aggregation pattern and possess unique supramolecular potentials, resulting from their distinct binding sites, such as tunability, compartmentalization of binding sites, and guest induced aggregation. ${ }^{21-25}$

In spite of their dynamic nature and great supramolecular potential bile salts still remain under-studied in several aspects - especially in terms of application as a green medium for chemical transformations. Therefore, we attempted to study the photocycloaddition of acenaphthylene in the medium for two primary reasons: (a) photochemistry of acenaphthylene has been used as a probe to gain insight into the mobility and immediate spatial environment of the encapsulating medium, (b) the reaction would also allow us to explore the bile salts aggregates as a green medium to substitute for organic solvents to perform photodimerization reactions. Herein, we report the outcome of our detailed studies exploring the interior of the bile salt aggregates using photochemistry of acenaphthylene as a probe.

\section{Results and Discussion}

\subsection{Photochemistry of Acenaphthylene}

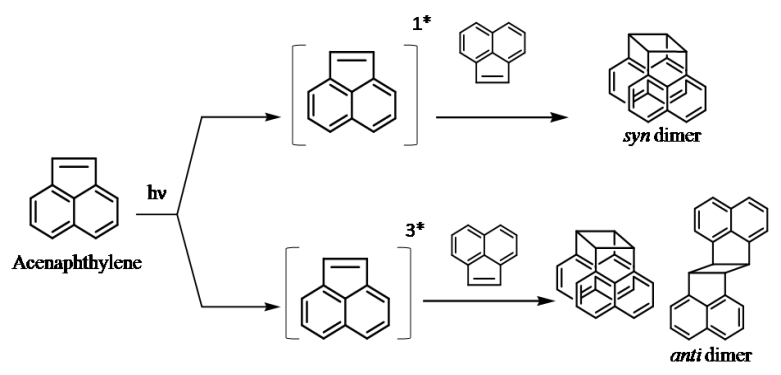

Scheme 1. Photochemistry of acenaphthylene

Acenaphthylene (ACN) yields two dimers upon irradiation that are stereo isomers (Scheme 1) - the syn and the anti. ${ }^{26,27}$ The proportion of syn and the anti dimers in the product mixture is dependent on three main factors, viz. multiplicity of excited state, concentration, and polarity of medium. Investigations conducted by Cowan et. al. ${ }^{26}$ led them to ascertain that acenaphthylene in its singlet $\left(\mathrm{S}_{1}\right)$ excited state yields the syn dimer while the triplet $\left(\mathrm{T}_{1}\right)$ state yields both anti dimer ( $\sim 90 \%)$ and syn dimer $(\sim 10 \%)$. With respect to concentration: higher concentration of acenaphthylene favors the formation of syn dimer over anti, and dilution results in the increase of anti dimer formation, which is in fact a consequence of the singlet-triplet dependent photochemistry; at high concentrations acenaphthylene has greater probability of reacting from the short lived singlet state to yield the syn dimer. At lower concentrations due to its shorter lifetime, the probability of $\mathrm{S}_{1}$ surviving long enough to encounter another acenaphthylene in the ground state is reduced. Hence the proportion of the syn dimer in the product mixture decreases resulting in the reduction of syn dimer proportion.

Due to the medium influenced photochemistry, ACN has been often utilized to understand the dynamics of encapsulated guest in unexplored medium. ${ }^{28-32}$ Under the same consideration, we have performed the photochemistry of ACN in $\mathrm{NaCh}$ to understand the nature of the medium.

In order to understand the nature of the bile salt micelles as a homogeneous medium and the variation in supramolecular characteristics of the aggregates with changes in concentration, the photochemistry of ACN was explored by observing product distribution with changes in concentration of sodium cholate.

\subsection{Inclusion of Acenaphthylene within Sodium Cholate Aggregates}

The procedure for complexation of guest involves initial sonication of mixture of sodium cholate and acenaphthylene in water, followed by even stirring of solution for six hours. Solubilization of guest within the aggregates is easily noted by observing increasing solution homogeneity over time. Evidence of guest included within aggregates may be obtained by recording ${ }^{1} \mathrm{H}$ NMR of an identical solution prepared in $\mathrm{D}_{2} \mathrm{O}$ (Figure 3 ) where aromatic guest signals could be seen alongside those of host's aliphatic signals. Acenaphthylene does not dissolve in water to any detectable extent in NMR even after sonication followed by stirring for extended durations.

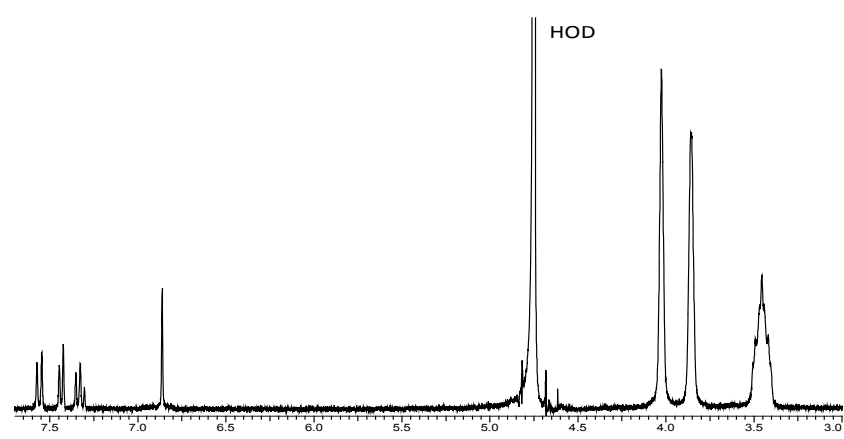

Figure 3. Partial ${ }^{1} \mathrm{H}$ NMR spectrum of acenaphthylene $(0.66 \mathrm{mM})$ solubilized in $\mathrm{D}_{2} \mathrm{O}$ by sodium cholate aggregates $(45 \mathrm{mM})$ shows the aggregate mediated dissolution of guest in aqueous medium

\subsection{Photoreactivity of Acenaphthylene in Sodium Cho- late}

Table 1 presents the product distribution resulting from the irradiation of solutions in which concentration of $\mathrm{NaCh}$ was varied incrementally ( $5 \mathrm{mM}$ to $250 \mathrm{mM}$ ) while that of the guest ACN was maintained at $0.66 \mathrm{mM}$. The concentra- 
tion range for $\mathrm{NaCh}$ in the experiments were chosen to entail equilibrium situations in which the predominant equilibrium species shift from $\mathrm{NaCh}$ monomer (at lower concentrations) to primary aggregates to secondary aggregate (at higher concentrations).

Table 1. Product selectivity observed in photochemistry of acenaphthylene $(\mathrm{ACN})$ incorporated in presence of varying amounts of sodium cholate in aqueous sodium chloride solution

\begin{tabular}{|c|c|c|c|c|}
\hline $\begin{array}{c}\text { [NaCh] } \\
\mathbf{m M}\end{array}$ & $\begin{array}{c}\text { Predominant aggre- } \\
\text { gate type }^{15}\end{array}$ & $\begin{array}{c}\mathbf{\%} \\
\text { syn }\end{array}$ & $\begin{array}{c}\mathbf{\%} \\
\text { anti }\end{array}$ & syn:anti \\
\hline 5 & Monomer-Primary & 66 & 34 & 1.94 \\
\hline 20 & Primary & 71 & 29 & 2.45 \\
\hline 40 & Primary-Secondary & 78 & 22 & 3.55 \\
\hline 90 & Secondary & 84 & 16 & 5.25 \\
\hline 140 & Secondary & 87 & 13 & 6.69 \\
\hline 190 & Secondary & 86 & 14 & 6.14 \\
\hline 250 & Secondary & 88 & 12 & 7.33 \\
\hline
\end{tabular}

Note: Concentration of acenaphthylene was fixed at $0.66 \mathrm{mM} .25 \mathrm{mM}$ sodium chloride aqueous solution was used to prepare sodium cholate solution. Conversion in all cases was between $30-40 \%$. Entries are average of three independent experiments.

Though the formal $\mathrm{CMC}$ of $\mathrm{NaCh}$ is commonly accepted to be between $15 \mathrm{mM}$ and $21 \mathrm{mM},{ }^{33-36}$ unlike straight chain surfactants, it has been established in literature that bile salts form micelles at concentrations well below the CMC. The same fact was corroborated in our studies where we observed that ACN $(0.66 \mathrm{mM})$ was in fact solubilized by $\mathrm{NaCh}$ at concentration as low as $5 \mathrm{mM}$. The solution, upon irradiation resulted in the formation of $66 \%$ syn dimer and $34 \%$ anti dimer. Whereas, irradiation of the same amount of ACN solubilised in $20 \mathrm{mM} \mathrm{NaCh}$, the concentration in which the equilibrium predominantly lies towards primary aggregates, the medium favored the formation of syn dimer by $71 \%$ over $29 \%$ for anti. The trend of increase in syn dimer proportion with increase in concentration of bile salt persisted as the concentration of $\mathrm{NaCh}$ was increased further to $40 \mathrm{mM}, 90 \mathrm{mM}$, and $140 \mathrm{mM}$. At $140 \mathrm{mM}$ of $\mathrm{NaCh}$, $87 \%$ of syn and $13 \%$ of anti dimer were observed, and at concentrations higher than $90 \mathrm{mM}$ there were no significant changes in the syn/anti proportions.

Table 2. Product distribution obtained from the irradiation of varying amounts acenaphthylene in presence of a constant concentration of sodium cholate ${ }^{\mathrm{a}}$ in water

\begin{tabular}{|c|c|c|c|}
\hline$[\mathbf{A C N}] \mathbf{~ m M}$ & \% syn & \% anti & syn:anti \\
\hline 0.66 & 83 & 17 & 4.26 \\
\hline 1.5 & 80 & 20 & 4.88 \\
\hline 2.5 & 81 & 19 & 5.25 \\
\hline 3.5 & 83 & 17 & 3.34 \\
\hline 4.5 & 82 & 18 & 4.26 \\
\hline
\end{tabular}

Note: Concentration of sodium cholate was maintained at $45 \mathrm{mM} .25 \mathrm{mM}$ sodium chloride aqueous solution was used to prepare sodium cholate solution. The conversion in all cases was between $30-40 \%$.

Table 2 presents the results of photochemistry of ACN performed in which the concentration of the host was maintained constant at $45 \mathrm{mM}$ while that of the guest was varied between $0.66 \mathrm{mM}$ and $4.5 \mathrm{mM}$. Considering an average aggregation number of 6 for the primary aggregates, the mean occupancy numbers for the aforementioned concentrations vary between 0.09 and 0.6 . There were no significant changes in product distribution for the seven fold increase in guest concentration. The correlation between dimer proportion and $\mathrm{NaCh}$ concentration is represented as a plot in Figure 4.

Control studies were also performed to serve as reference points to help understand the effect of spin multiplicities in the product distribution within the medium (Table 3). Irradiations were performed in $\mathrm{O}_{2}$ purged solution, a triplet quencher, resulted in $94 \%$ syn dimer - $11 \%$ higher formation of syn dimer compared to the same in $\mathrm{N}_{2}$ purged solution.

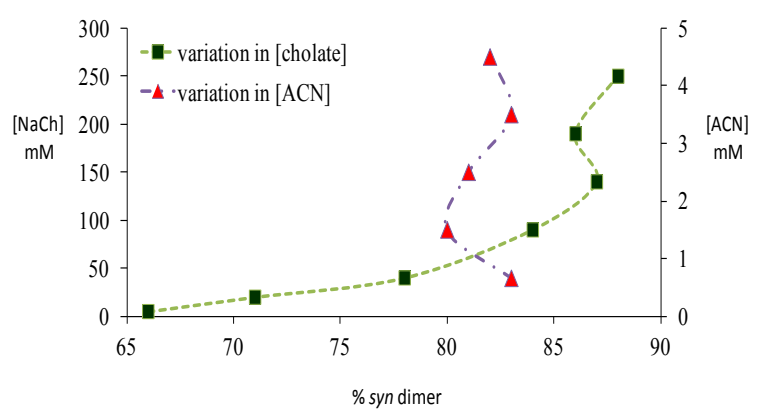

Figure 4. Photoproduct distribution from reaction of $\mathrm{ACN}$ and variation with concentration changes

When Eosin- $Y$, a triplet sensitizer, was added to the solution of ACN solubilised in $\mathrm{NaCh}, 56 \%$ syn dimer resulted with anti dimer still being the minor product. When a solution of identical concentration of ACN in hexane was irradiated in presence of Eosin- $\mathrm{Y}$, the anti dimer was the major product.

Table 3. Product distribution obtained from the irradiation of varying amounts acenaphthylene in presence of a constant concentration of sodium cholate $^{\mathrm{a}}$ in water

\begin{tabular}{|c|c|c|c|c|}
\hline Medium & Condition & Role & \% syn & \% anti \\
\hline $\mathrm{NaCh}$ & $\mathrm{N}_{2}$-purged & & 83 & 17 \\
\hline $\mathrm{NaCh}$ & $\mathrm{O}_{2}$-purged & Triplet quencher & 94 & 6 \\
\hline $\mathrm{Hexane}$ & Eosin-Y & Triplet sensitizer & 45 & 55 \\
\hline $\mathrm{NaCh}$ & Eosin-Y & Triplet sensitizer & 56 & 44 \\
\hline
\end{tabular}

a) Concentration of acenaphthylene $1.3 \mathrm{mM}$ and that of $\mathrm{NaCh} 45 \mathrm{mM}$. b) Concentration of Eosin-Y was approximately $10^{-5} \mathrm{M}$. The solutions were irradiated with a $410 \mathrm{~nm}$ cut-off filter.

\subsection{Discussion of Results}

Selectivity in product distribution of acenaphthylene in constrained medium is shown to be primarily controlled by two factors: the spin states of ACN in the excited state, ${ }^{21,22}$ and the availability of space, where spatial limitations favor the more compact syn dimer over anti. ${ }^{28}$ The possibility of a ground state complex of acenaphthylene preoriented to form the syn dimer could be ruled out based on the reports by Bohne et. al. ${ }^{27}$ as this would require multiple occupancy for ACN within the primary aggregates of sodium cholate primary binding sites are too small to accommodate two guest molecules simultaneously.

To gauge the influence of the spatial factor alone on the 
product distribution, the contribution from the spin factor was eliminated by using triplet quenchers and sensitisers (Table 3). In the absence of spin state manipulating species, ACN reacts to yield $83 \%$ syn and $17 \%$ anti dimer. Whereas, the same for oxygen purged solution (oxygen being a triplet quencher) yielded almost exclusively syn dimer. The formation of as much as $94 \%$ of syn dimer in the medium is indicative of the fact that the medium does not thwart the near quantitative formation of syn dimer.

On the other hand, when the reaction was performed with Eosin- $Y$ in the medium, a triplet sensitizer, yielded $56 \%$ syn dimer; comparing this with the Eosin-Y sensitized reaction in hexane where no spatial constraints exist, $11 \%$ more syn dimer (Table 3) was obtained within the aggregates. Considering the results from control studies presented in table 3, it is evident that the formation of syn dimer is favored within the primary aggregates most likely due to its compactness in shape, as the space available for binding within the aggregates is both limited and very rigid.

The notable feature in the photodimerization of acenaphthylene within in sodium cholate micelles is the observed trend of increase in syn dimer formation within increase in micelle concentration until $90 \mathrm{mM}$ and no significant changes thereafter (Figure 4). By correlating the bile salt equilibrium dynamics with the trend in syn:anti proportion in reaction outcome with changes in $\mathrm{NaCh}$ concentration, it is apparent that as the concentration of bile salt increases, the equilibrium shifts towards the secondary aggregates formation the photochemistry of acenaphthylene is steered towards the formation of syn dimer in higher proportion.

In order to explain the observed trend in product distribution, we propose the following: acenaphthylene, being highly nonpolar, is expected to be solubilised in primary aggregates only. At low concentrations of $\mathrm{NaCh}$ secondary aggregates is expected to be absent and, therefore, ACN will be solubilised mostly by primary aggregates. In this situation, the probability of dimerization of $A C N$ in its $S_{1}$ and $T_{1}$ states are limited by the diffusion of the primary aggregates - the mobility of the guest is only as fast as the mobility of the primary aggregate itself. Since the $S_{1}$ state is short-lived than the $T_{1}$ state (by three orders of magnitude) the low $\mathrm{NaCh}$ concentration situation favors reactivity from the $T_{1}$ state more than the $S_{1}$ as the latter would be the first to "die out" due to relaxation.

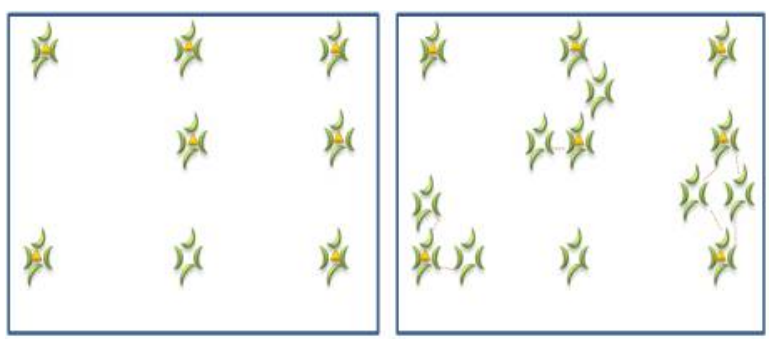

Figure 5. Pictorial representation of two different scenarios - low concentration (left) and high concentration (right) of $\mathrm{NaCh}-$ to explain the variation in product distribution obtained from the photochemistry of $\mathrm{ACN}$
On the other hand, at higher concentrations of $\mathrm{NaCh}$ the equilibrium shifts towards the formation of secondary aggregates which are conglomerates of primary aggregates. The secondary aggregates could be envisioned as a 'micro-environment of micellar network' (depicted in Figure 5) of primary aggregates loosely held together by forces of hydrogen-bonding interaction between the hydroxyl groups of bile salt and water molecules. Within this 'micellar network' the local mobility of solubilised guest molecule such as ACN would be significantly enhanced as multiple primary aggregates are held close to each other with the $\mathrm{NaCh}$ monomers engaged in the dissociation-association equilibrium. Due to enhanced mobility the probability of apolar guest solubilised within the primary aggregates, the probability of ACN reacting from its singlet excited state is greatly increased as the mobility of ACN is no longer limited to the diffusion of the primary aggregates only. This in turn would reflect in the increased formation of syn dimer as is the case.

This model explains the observed increase in syn dimer formation with increasing bile salt concentration until the concentration reaches $140 \mathrm{mM}$. For concentration increase beyond $140 \mathrm{mM}$ there appears to be no concomitant change in supramolecular characteristics of the aggregates. Based on the fact that there is no significant increase in the formation of syn dimer beyond $140 \mathrm{mM}$, we believe that $140 \mathrm{mM}$ is the concentration at which the equilibrium attributes in favour of secondary aggregates are at their highest. We also infer that increasing concentration of $\mathrm{NaCh}$ beyond 140 $\mathrm{mM}$ does not enrich the medium with secondary aggregates at the same rate observed between the $20 \mathrm{mM}-140 \mathrm{mM}$ bracket. In order to confirm that it is the concentration of secondary aggregates and the proximity of the primary aggregates within the secondary aggregates that affects the photoproduct distribution of acenaphthylene photodimers, we performed experiments in which concentration of ACN was varied while that of Nach was maintained at $45 \mathrm{mM}$ (Table 2). In this case, as expected, not much variation in product distribution was observed which substantiates the hypothesis that at lower concentrations of $\mathrm{NaCh}$, the reactivity is limited by the diffusion of primary aggregates. The clear influence of sodium cholate assembly equilibrium on the photochemical outcome of acenaphthylene is evident from the trend presented in figure 4, where syn:anti ratio changes with $\mathrm{NaCh}$ concentration. Our studies have lead us to understand the dynamic nature of the bile salt aggregates and the mobility of guest molecule within the assembly.

\section{Conclusions}

Sodium cholate aggregates were employed to perform the photochemical dimerization of acenaphthylene. Concentration variation experiments and control experiments were performed to understand the interior of the primary aggregates and the shift in supramolecular characteristics of the bile salt micro-heterogeneous medium. Results indicate that 
the tight spaces within primary aggregates favor the formation of syn dimer over the spatially demanding anti dimer. In addition the concentration dependent photochemistry suggests that at lower concentrations of $\mathrm{NaCh}$ the mobility of the guest is limited by the diffusion characteristics of the primary aggregates. Whereas, at higher concentration of $\mathrm{NaCh}$ the predominance of secondary aggregates act as a micro-network of primary aggregates increasing the mobility of the guest molecules. The reaction provides insight into the mobility and spatial constraint of apolar guest molecules encapsulated within the bile salt micellar system.

\section{Experimental}

\section{Materials}

Sodium cholate $(\mathrm{NaCh})$, sodium chloride, anhydrous sodium sulfate, and eosin-Y, were procured from Sigma Aldrich Co. and used without further purification. Acenaphthylene procured from Sigma Aldrich Co. was 95\% acenaphthylene and 5\% acenaphthene as indicated in the manufacturer's label and analyzed by us in the GC. The compound was purified by vacuum sublimation technique during which acenaphthylene sublimed on the upper walls of the sublimator while acenaphthene remains at the bottom. This procedure was repeated to obtain acenaphthylene more than $98 \%$ pure.

Mass Balance Experiments:

Mass balance experiments were carried out to confirm that product selectivities observed were not due to selective decomposition, loss, inclusion, or exclusion of reaction components. A standard solution with equal amounts of the acenaphthylene and an external standard $(2 \mathrm{mg}$ each in $5 \mathrm{~mL}$ of hexane-ethyl acetate) for GC (naphthalene) was prepared and injected in the GC. The ratio of the peak intensities was taken as a standard for 1:1 mixture by mass. To determine the mass balance after photochemistry, the irradiated samples were extracted and mixed with an equal mass (as that of the reactive guest taken) of internal standard and analyzed in the GC. The ratio of all peaks in the GC run with respect to the internal standard was considered to calculate the mass balance. In all cases the mass balance was estimated to be at least $80 \%$.

Complexation, irradiation and extraction procedure:

A standard solution of acenaphthylene $(5 \mathrm{mg} / \mathrm{mL})$ in dichloromethane was prepared. Volume corresponding to 0.5 $\mathrm{mg}$ was pipetted out and solvent evaporated to obtain a thin film of the compound. $20 \mathrm{~mL}$ of $25 \mathrm{mM}$ sodium chloride solution, $45 \mathrm{mg}$ of sodium cholate were added to the acenaphthylene thin film. Sonication for 15 minutes and allowing the solution to stir for 2 hours resulted in formation of clear yellow colored solutions. The solutions were filtered with a Whatmann filter paper of fine porosity and slow flow rate. The filtrate was purged with nitrogen for 15 minutes, sealed and irradiated with a $410 \mathrm{~nm}$ cut-off filter. The cut-off filter was used to prevent dimers from absorbing any radiation from the photochemical lamp source.
After irradiation, the solution was diluted by adding 30 $\mathrm{mL}$ of deionized water. Extraction of the starting material and reactants was performed in a separatory funnel with 30 $\mathrm{mL}$ of ethyl acetate and $15 \mathrm{~mL}$ of acetonitrile mixture. The organic layer was dried over anhydrous sodium sulfate, and analyzed in a Shimadzu model 4020 GC-MS fitted with an Agilent SE-30 column. The identities of the dimers were also established by ${ }^{1} \mathrm{H}$ NMR spectroscopy.

Reported values

All values reported in tables are averages of three independently performed experiments. The averages are rounded off to the closest unit and the error limit for reported values is $\pm 3 \%$.

Analysis of photodimers of acenaphthylene:

The identities of the photoproducts were confirmed by NMR spectroscopy recorded on a Bruker Avance $300 \mathrm{MHz}$ spectrometer and referenced using the residual solvent signal as the internal standard. The chemical shifts were matched with those reported in literature. ${ }^{22}$

Syn dimer: ${ }^{1} \mathrm{H}$ NMR $\left(\mathrm{CDCl}_{3}\right) \delta 7.18(\mathrm{~d}, 4 \mathrm{H}, J=8.0 \mathrm{~Hz})$, $7.15(\mathrm{~d}, 4 \mathrm{H}, J=7.5 \mathrm{~Hz}), 7.03(\mathrm{~d}, 4 \mathrm{H}, J=7.3 \mathrm{~Hz}), 4.85$ (s, $4 \mathrm{H})$.

Anti dimer: ${ }^{1} \mathrm{H}$ NMR $\left(\mathrm{CDCl}_{3}\right) \delta 7.74(\mathrm{~d}, 4 \mathrm{H}, \mathrm{J}=8 \mathrm{~Hz})$, $7.60(\mathrm{~d}, 4 \mathrm{H}, \mathrm{J}=7.9 \mathrm{~Hz}), 7.01(\mathrm{~d}, 4 \mathrm{H}, \mathrm{J}=7.9 \mathrm{~Hz}), 4.11(\mathrm{~s}$, $4 \mathrm{H})$.

For routine analysis and quantitative determination of products and reactant composition in the reaction mixture, samples were analyzed as such after extraction in a GC-MS.

GC-MS program: Initial temp $100^{\circ} \mathrm{C}$, Initial time 1 minute, Initial rate $10^{\circ} \mathrm{C} /$ minute, final temperature $300^{\circ} \mathrm{C}$ and final time 5 minutes. Retention times: ACN at $7.5 \mathrm{~min}$, syn dimer at $13.3 \mathrm{~min}$ and anti dimer at $16.1 \mathrm{~min}$.

\section{ACKNOWLEDGEMENTS}

The work presented in the manuscript was performed with the financial support from VP's office at WNMU. MP thanks Mr. Ross Fischer in the Dept. Of Natural Sciences, WNMU for his support, and Dr. Gopalan (New Mexico State University, Las Cruces) for helping us in acquiring NMR spectra for characterization. MP is very thankful to Dr. V. Ramamurthy for providing insight and advice in this project.

\section{REFERENCES}

[1] A. Singh, J.D. VanHamme, and O.P. Ward, Biotechnol. Adv., 2007, 25 (1), 99.

[2] A.S. Yazdi, Trends Anal. Chem., 2011, 30 (6), 918.

[3] A.N. Lukyanov, and V.P. Torchilin, Adv. Drug Delivery Rev., 2004, 56 (9), 1237.

[4] E. Semo, E. Kesselman, D. Danino, Y. D. Livney, Food Hydrocolloids, 2007, 21, 5-6, 936. 
[5] S. Paria, Adv. Colloid Interface Sci., 2008, 138 (1), 24.

[6] S. Kim, Y. Shi, J. Kim, K. Park, and J. Cheng, Expert Opin. Drug Discovery, 2010, 7 (1), 49.

[7] K. Trickett, and J. Eastoe, Adv. Colloid Interface Sci., 2008, 144 (1-2), 66.

[8] L. Kumar, T. Mahajan, and D. D. Agarwal, Ind. Eng. Chem. Res.2012, 52 (5), 2227.

[9] M. Pattabiraman, L.S. Kaanumalle, and V. Ramamurthy, Langmuir, 2006, 22 (5), 2185.

[10] S. Bhat, and U. Maitra, Molecules, 2007, 12(9), 2181.

[11] F. Jiang, J. Du, X. Yu, J. Bao, and X. Zeng, J. Colloid Interface Sci., 2004, 210 (2), 497.

[12] U. Tonellato, Pure Appl. Chem., 1998, 70(10), 1961.

[13] F. Trentin, A. Scarso, and G. Strukul, Tetrahedron Lett. 2011, $52(51), 6978$

[14] A. Schreiber, F. R. Simon, J, Pediatr. Gastroenterol. Nutr. 1983, 2(2), 337.

[15] P. Mukerjee, Y. Moroi, M. Murata, and A. Y. S. Yang, Hepatology, 1984, 4 (5 suppl), 61s.

[16] P. Mukerjee, and J. R. Cardinal, J. Pharm. Sci. 1976, 65 (6), 882.

[17] P. Ekwall, J. Colloid Sci. 1954, (Suppl. 1), 66.

[18] L. Hao, R. Lu, D.G. Leaist, and P. R. Poulin, J. Soln. Chem. 1997, 26 (2), 113.

[19] D.M. Small, In The Bile Salts; P.P. Nair, D. Kritchevsky, Eds.; Plenum Press: New York, 1971, Vol. 1, pp 249-256.

[20] D.M Small, S.A. Penkett, and D. Chapman, Biochim. Biophys. Acta, 1969, 176 (1), 178.

[21] R. Li, E. Carpentier, E. D. Newell, L. M. Olague, E. Heafey,
C. Yihwa, and C. Bohne, Langmuir. 2009, 25 (24), 13800.

[22] G. Conte, R. Di Blasi, E. Giglio, A. Parretta and N. V. Pavel, J. Phys. Chem., 1984, 88 (23), 5720.

[23] A. Jover, F. Meijide, E. Rodríguez Núñez and J. Vásquez Tato, M. Mosquera. Langmuir, 1997, 13 (2), 161.

[24] L. L. Amundson, R. Li, and C. Bohne, Langmuir, 2008, 24 (16), 8491.

[25] C. Ju, and C. Bohne, Photochem. Photobiol. 1996, 63 (1), 60.

[26] D. O. Cowan, and R. L. E. Drisko, J. Am. Chem. Soc. 1970, 92 (21), 6286 .

[27] N. Haga, H. Takayanagi, and K. Tokumaru, J. Org. Chem.1997, 62 (11), 3734.

[28] M. Yoshizawa, Y. Takeyama, T. Okano, and M. Fujita. J. Am. Chem. Soc. 2003, 125 (11), 3243.

[29] V. Ramesh, and V. Ramamurthy, J. Photochem. 1984, 24 (4), 35.

[30] L. S. Kaanumalle, and V. Ramamurthy, Chem. Commun. 2007, (10), 1062.

[31] D. Madhavan, K. Pitchumani. Photochem. Photobiol. Sci. 2003, 2(2), 95 .

[32] Y. Matsuo, T. Fukunaga, N. Tokura, T. Fukutsuka, and Y. Sugie. Trans. Mat. Res. Soc. Jpn. 2003, 28(3), 589.

[33] L. B. Pa'rtay, M. Sega, and P. Jedlovszky, Langmuir, 2007, 23 (24), 12322.

[34] P. Perez-Tejeda, R. Risquez, A. R. Perez, A. Terriza, and M. P. Leon. All Res. J. Chem. 2010, 1 (1), 13.

[35] A. Coello, F. Meijide, E. R. Nunez, and J. V. Tato, J. Pharm. Sci. 1996, 85(1), 9.

[36] S. Reis, C. G. Moutinho, C. Matos, B. de Castro, P. Gameiro, J. L. F. Lima, Anal. Biochem. 2004, 334, 117-126. 